\title{
ON THE LAW OF LARGE NUMBERS FOR DEMAZURE MODULES OF $\widehat{\mathfrak{s l}}_{2}$
}

\author{
THOMAS BLIEM AND STAVROS KOUSIDIS
}

\begin{abstract}
We determine the covariance of the weight distribution in level 1 Demazure modules of $\widehat{\mathfrak{s l}}_{2}$. This allows us to prove a weak law of large numbers for these weight distributions, and leads to a conjecture about the asymptotic concentration of weights for arbitrary Demazure modules.
\end{abstract}

\section{INTRODUCTION}

The motivation for this work is to understand the qualitative features of dimensions of weight spaces in Demazure modules $V_{w}(\Lambda)$ as the highest weight $\Lambda$ is fixed and the length of the Weyl group element $w$ becomes large. We want to be able to answer questions like: Where is the bulk of weights, counted with their individual multiplicities, concentrated? Do they tend to spread out or do they concentrate in a certain part of the Weyl polytope? Etc.

Even though there is a large body of work on Demazure modules, these kind of questions are still difficult to answer. We believe that in order to answer them, one needs to look at weight distributions with a probabilistic eye, starting with the computation of the expected value and the covariance, possibly followed by skewness and kurtosis later. We determined the expected value of the weight distribution of any Demazure module of $\widehat{\mathfrak{s l}}_{2}$ in [2]. The next step is to determine the covariance, which we complete in the present article for the modules in level 1:

Theorem 7.1. Let $j \in\{0,1\}$ and $w \in W^{\text {aff }}$ such that the length $l\left(w s_{j}\right)<l(w)=N$. Then the covariance matrix $\Sigma$ of the degree $\langle-d, \cdot\rangle$ and the finite weight $\left\langle\alpha_{1}^{\vee}, \cdot\right\rangle$ in $V_{w}\left(\Lambda_{j}\right)$ is given by

$$
\Sigma=\left\{\begin{array}{cc}
\left(\begin{array}{cc}
\frac{N(N-1)(2 N+5)}{96} & 0 \\
0 & N
\end{array}\right) & \text { if } N \equiv j \bmod (2), \\
\frac{N(N-1)(2 N+5)}{96}+\frac{N}{4} & \frac{N}{2} \\
\frac{N}{2} & N
\end{array}\right) \quad \text { if } N \not \equiv j \bmod (2) .
$$

See Figure 1 for some examples. Our Theorem 7.1 is enough to obtain the weak law of large numbers as a corollary, to our knowledge the first result to give an idea about the overall weight distribution in Demazure modules as the length of the Weyl group element becomes large:

2010 Mathematics Subject Classification. Primary 06B15 Representation theory, Secondary 60B99 Probability theory on algebraic and topological structures.

Key words and phrases. affine Kac-Moody algebra, Demazure module, variance, covariance, law of large numbers. 


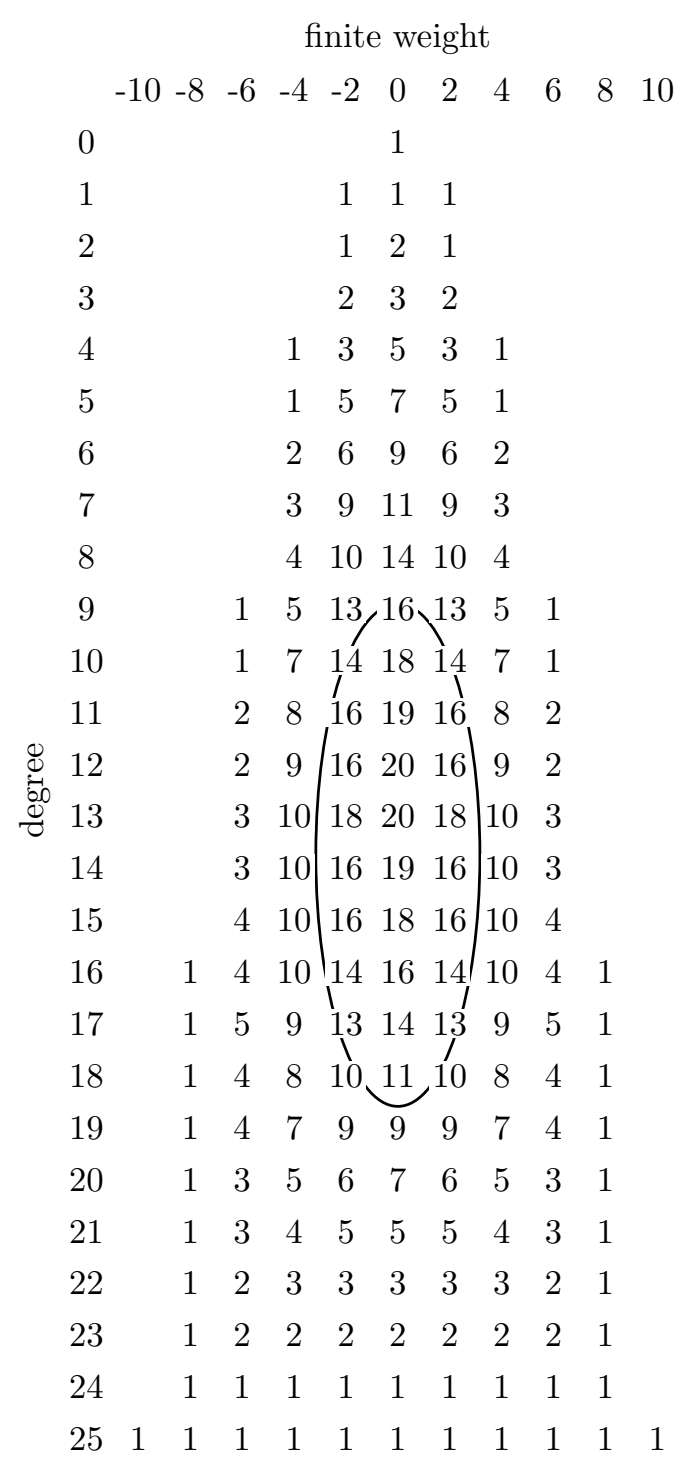
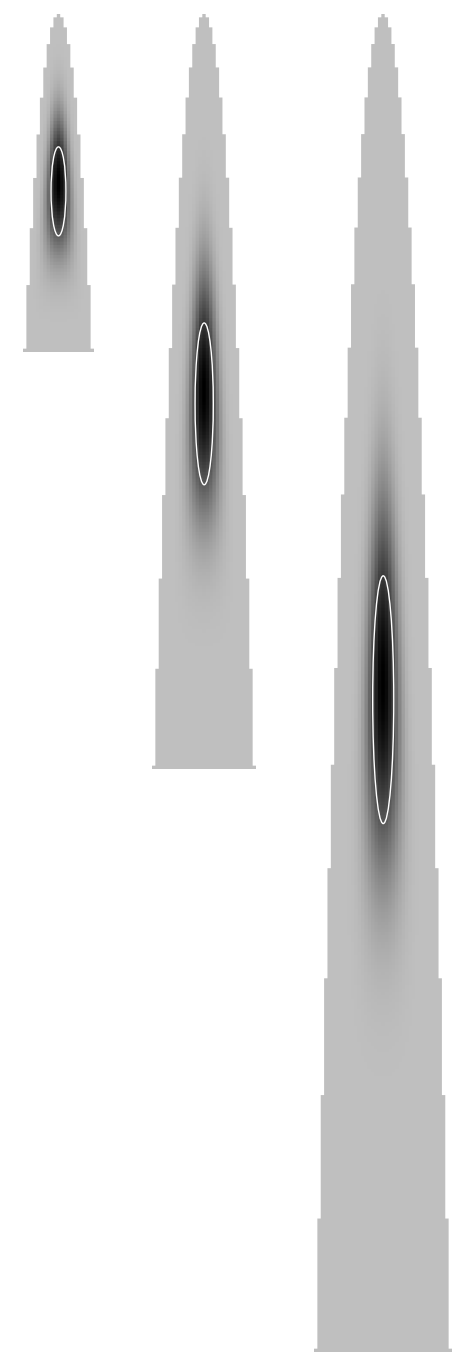

FIGURE 1. Weight distribution of $V_{\left(s_{1} s_{0}\right)^{k}}\left(\Lambda_{0}\right)$ for $k=5$ (indicated by numbers) and $k=10,15,20$ (indicated by shades of gray). The covariances are represented by covariance ellipses.

Theorem 8.2. Let $\left(\Lambda^{(k)}\right)$ be a sequence in $\left\{\Lambda_{0}, \Lambda_{1}\right\}$, and $\left(w^{(k)}\right)$ a sequence in $W^{\text {aff }}$ such that $l\left(w^{(k)}\right) \rightarrow \infty$. Let $\tilde{\mu}^{(k)} \in \operatorname{Meas}\left(\mathbf{R}^{2}\right)$ be the joint distribution of the degree and the finite weight in $V_{w^{(k)}}\left(\Lambda^{(k)}\right)$, normalized to a probability distribution and rescaled individually in the two coordinates such that $\operatorname{supp}\left(\tilde{\mu}^{(k)}\right)$ just fits into the rectangle $[0,1] \times[-1,1]$. Then, as $k \rightarrow \infty$,

$$
\tilde{\mu}^{(k)} \stackrel{\mathrm{w}}{\longrightarrow} \delta_{\left(\frac{1}{2}, 0\right)} .
$$




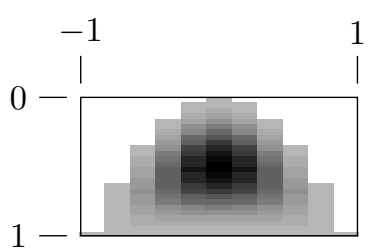

1

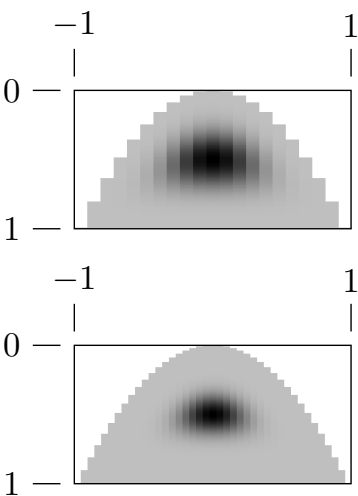

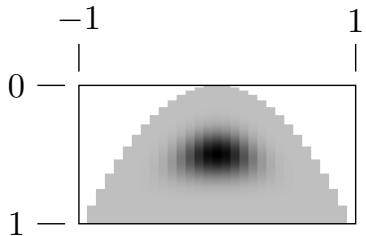

Figure 2. Weight distributions of $V_{\left(s_{1} s_{0}\right)^{k}}\left(\Lambda_{0}\right)$ for $k=5,10,15,20$, rescaled into the rectangle $[0,1] \times[-1,1]$.

Some examples of $\tilde{\mu}^{(k)}$ are shown in Figure 2. Furthermore, based on our present result and [2, Corollary 4.3] we propose the following conjecture:

Conjecture 8.3. Fix a dominant integral weight $\Lambda$ and a sequence $\left(w^{(k)}\right)$ in $W^{\text {aff }}$ such that $l\left(w^{(k)}\right) \rightarrow \infty$. Let $\mu^{(k)} \in \operatorname{Meas}(\mathbf{N} \times \mathbf{Z})$ be the joint distribution of the degree and the finite weight in $V_{w^{(k)}}(\Lambda)$. Let $\tilde{\mu}^{(k)} \in \operatorname{Meas}\left(\mathbf{R}^{2}\right)$ be the distribution obtained from $\mu^{(k)}$ by normalizing to a probability distribution and rescaling the two coordinates individually so that $\operatorname{supp}\left(\tilde{\mu}^{(k)}\right)$ just fits into the rectangle $[0,1] \times[-1,1]$. Then, as $k \rightarrow \infty$,

$$
\tilde{\mu}^{(k)} \stackrel{\mathrm{w}}{\longrightarrow} \delta_{\left(\frac{\langle c, \Lambda\rangle+2}{3(\langle c, \Lambda\rangle+1)}, 0\right)},
$$

where $c=\alpha_{0}^{\vee}+\alpha_{1}^{\vee}$ denotes the canonical central element.

The outline of the proof of Theorem 7.1 and the organization of the paper is as follows: It is easy to show that the degree and the finite weight are uncorrelated. The covariance of the finite weight can be directly obtained from a known result by Sanderson about the real characters of Demazure modules [10]. Hence the main part is about the variance of the degree distribution. The strategy is to proceed by induction along Demazure's character formula. In \$3, we show that a single recursion step expresses the second moment of the degree distribution of a given Demazure module in terms of the third moments of the weight distribution of a smaller Demazure module. We try to express these third moments in terms of the (known) third moments of the distribution of the finite weight, but succeed not quite (Proposition 3.1). In $\$ 4$, we show that the weight multiplicities are symmetric in each string of weights differing only by a multiple of the null root $\delta$ Lemma 4.1). We use this in $\$ 5$ to show that the covariance between two specific quadratic functions vanishes (Corollary 5.4). This allows us to explicitly compute the previously problematic third moments (Lemma 5.5). This yields an explicit recurrence relation of a purely additive nature (Lemma 6.2), which is easy to solve Corollary 6.3. We complement our result in $\$ 7$ by determining the full covariance matrix (Theorem 7.1). In $\S 8$ we deduce a weak law of large numbers for our Demazure modules (Theorem 8.2) and formulate a conjecture for the general case Conjecture 8.3. 


\section{Notation}

For general notation about Kac-Moody algebras we mostly follow Kac [4. Let $\mathfrak{h}$ be a 3-dimensional complex vector space and $\alpha_{0}^{\vee}, \alpha_{1}^{\vee} \in \mathfrak{h}, \alpha_{0}, \alpha_{1} \in \mathfrak{h}^{*}$ a realization of the generalized Cartan matrix $A=\left(\begin{array}{cc}2 & -2 \\ -2 & 2\end{array}\right)$. Let $\widehat{\mathfrak{s l}}_{2}=\mathfrak{g}(A)$ be the associated affine Lie algebra. We denote the canonical pairing between $\mathfrak{h}$ and $\mathfrak{h}^{*}$ by $\langle h, \alpha\rangle=\alpha(h)$. Choose $d \in \mathfrak{h}$ such that $\left\langle d, \alpha_{0}\right\rangle=1$ and $\left\langle d, \alpha_{1}\right\rangle=0$. Such an element $d$ is called a scaling element. The degree of $\lambda \in \mathfrak{h}^{*}$ is defined as $\langle-d, \lambda\rangle$. The set $\left\{\alpha_{0}^{\vee}, \alpha_{1}^{\vee}, d\right\}$ is a basis of $\mathfrak{h}$. Let $\left\{\Lambda_{0}, \Lambda_{1}, \delta\right\}$ be the corresponding dual basis of $\mathfrak{h}^{*}$. Then $\delta=\alpha_{0}+\alpha_{1}$, and $\Lambda_{0}, \Lambda_{1}$ are called fundamental weights.

Integrable highest weight modules of $\widehat{\mathfrak{s l}}_{2}$ are parametrized up to isomorphism by dominant integral weights

$$
\Lambda=m \Lambda_{0}+n \Lambda_{1}+z \delta
$$

for $m, n \in \mathbf{N}$ and $z \in \mathbf{C}$. We denote the integrable highest weight module corresponding to $\Lambda$ by $V(\Lambda)$. As a change in $z$ simply corresponds to the choice of a different scaling element $d \in \mathfrak{h}$, it is customary to suppose $z=0$ and only consider dominant integral weights of the form $\Lambda=m \Lambda_{0}+n \Lambda_{1}$, which we do from now on.

Let $c=\alpha_{0}^{\vee}+\alpha_{1}^{\vee}$ be the canonical central element. The level of a weight $\lambda \in \mathfrak{h}^{*}$ is defined as $\langle c, \lambda\rangle$. If $\lambda=m \Lambda_{0}+n \Lambda_{1}$, then its level is $\langle c, \lambda\rangle=m+n$. Hence the dominant integral weights of level 1 are exactly the fundamental weights $\Lambda_{0}, \Lambda_{1}$.

A weight $\lambda \in \mathfrak{h}^{*}$ is said to occur in a given integrable highest weight module $V(\Lambda)$ if the weight space $V(\Lambda)_{\lambda}$ is nontrivial. The set of weights occuring in $V(\Lambda)$ is contained in the (affine) lattice

$$
\Gamma=\Lambda+\mathbf{Z} \alpha_{0}+\mathbf{Z} \alpha_{1} .
$$

We define coordinates $a, b$ on $\Gamma$ by

$$
\lambda=\Lambda-a(\lambda) \alpha_{0}-b(\lambda) \alpha_{1}
$$

for all $\lambda \in \Gamma$. Note that $a, b$ depend on $\Lambda$. In Figure 1 each matrix component resp. pixel represents a point in $\Gamma$. We write $\Gamma_{j}=\Lambda_{j}+\mathbf{Z} \alpha_{0}+\mathbf{Z} \alpha_{1}$ for $j \in\{0,1\}$ to refer to the two lattices corresponding to the fundamental weights.

For $j \in\{0,1\}$ define linear maps $s_{j}: \mathfrak{h}^{*} \rightarrow \mathfrak{h}^{*}$ by

$$
s_{j}(\lambda)=\lambda-\left\langle\alpha_{j}^{\vee}, \lambda\right\rangle \alpha_{j} .
$$

The Weyl group $W^{\text {aff }}$ of $\widehat{\mathfrak{s l}}_{2}$ is by definition the subgroup of $\mathrm{GL}\left(\mathfrak{h}^{*}\right)$ generated by $s_{0}$ and $s_{1}$. All elements of $W^{\text {aff }}$ have the form

$$
w_{N, 0}=\underbrace{\cdots s_{0} s_{1} s_{0}}_{N \text { factors }} \quad \text { or } \quad w_{N, 1}=\underbrace{\cdots s_{1} s_{0} s_{1}}_{N \text { factors }}
$$

for $N \geq 0$. We abbreviate $w_{N}=w_{N, 0}$, as these are the elements we will mostly discuss. Write $l(w)$ for the length of a reduced decomposition of $w \in W^{\text {aff }}$, so $l\left(w_{N, j}\right)=N$.

Let $\mathfrak{n}_{+} \subset \widehat{\mathfrak{s l}}_{2}$ be the sum of the positive root spaces. For $w \in W^{\text {aff }}$ and $\Lambda$ a dominant integral weight, define the Demazure module $V_{w}(\Lambda)$ [7, 5, 8] to be the

${ }^{1}$ The software used to produce most of the figures in this article is available at http://sourceforge. net/projects/demazure 
$\left(\mathfrak{h} \oplus \mathfrak{n}_{+}\right)$-module generated by $V(\Lambda)_{w \Lambda}$. As $V_{w}(\Lambda)$ is in particular an $\mathfrak{h}$-module, it has a weight space decomposition

$$
V_{w}(\Lambda)=\bigoplus_{\lambda \in \mathfrak{h}^{*}} V_{w}(\Lambda)_{\lambda}
$$

Let $\operatorname{Meas}_{\mathrm{c}}(\Gamma)$ denote the set of measures on $\Gamma$ with compact (hence finite) support. We define the weight distribution of $V_{w_{N, j}}(\Lambda)$ to be

$$
\mu_{N, j}=\sum_{\lambda \in \mathfrak{h}^{*}} \operatorname{dim}\left(V_{w_{N, j}}(\Lambda)_{\lambda}\right) \cdot \delta_{\lambda} \in \operatorname{Meas}_{\mathrm{c}}(\Gamma)
$$

and again abbreviate $\mu_{N}=\mu_{N, 0}$ for the case mostly considered. Note that the dependence on $\Lambda$ is important, but only implicit in the notation.

Given $\Lambda$, define operators $D_{0}, D_{1}$ on the space $\operatorname{Meas}_{\mathrm{c}}^{ \pm}(\Gamma)$ of signed measures on $\Gamma$ with compact support by

$$
D_{j} \delta_{\lambda}=\sum_{i=0}^{\left\langle\alpha_{j}^{\vee}, \lambda\right\rangle} \delta_{\lambda-i \alpha_{j}}
$$

for $j \in\{0,1\}$ and $\lambda \in \Gamma$. Here we use the conventions that $\sum_{i=0}^{-1} a_{i}=0$ and $\sum_{i=0}^{k} a_{i}=-\sum_{i=k+1}^{-1} a_{i}$ for $k<-1$, hence the necessity to consider signed measures. Demazure's character formula for Kac-Moody algebras [7, 5, 8, states that

$$
\mu_{N, 0}=\underbrace{\cdots D_{0} D_{1} D_{0}}_{N \text { factors }} \delta_{\Lambda} \text { and } \mu_{N, 1}=\underbrace{\cdots D_{1} D_{0} D_{1}}_{N \text { factors }} \delta_{\Lambda} .
$$

Consider elements of $\mathfrak{h}$ as functions on $\mathfrak{h}^{*}$. We refer to the push-forward measure $(-d)_{*} \mu_{N, j}$ as the degree distribution, and to $\left(\alpha_{1}^{\vee}\right)_{*} \mu_{N, j}$ as the distribution of the finite weight of $V_{w_{N, j}}(\Lambda)$. Note that in terms of the coordinates $a, b$ we have that as functions on $\Gamma_{i}$

$$
-d=a \quad \text { and } \quad \alpha_{1}^{\vee}= \begin{cases}-2(a-b) & \text { if } j=0, \\ -2(a-b)+1 & \text { if } j=1 .\end{cases}
$$

Hence the degree distribution is $a_{*} \mu_{N, j}$, and the distribution of the finite weight is $(a-b)_{*} \mu_{N, j}$ up to translation and scaling.

Recall that the expected value of a function $f: \Gamma \rightarrow \mathbf{R}$ with respect to a nonzero measure $\mu \in \operatorname{Meas}_{\mathrm{c}}(\Gamma)$ is

$$
\mathrm{E}_{\mu}[f]=\frac{1}{\mu(\Gamma)} \sum_{\lambda \in \Gamma} \mu(\{\lambda\}) f(\lambda) .
$$

The covariance of two functions $f$ and $g$ is

$$
\operatorname{Cov}_{\mu}(f, g)=\mathrm{E}_{\mu}\left[\left(f-\mathrm{E}_{\mu}[f]\right)\left(g-\mathrm{E}_{\mu}[g]\right)\right],
$$

and the variance of $f$ is $\operatorname{Var}_{\mu}(f)=\operatorname{Cov}_{\mu}(f, f)$.

\section{Almost a ReCURsion formula}

The characters of Demazure modules for the highest weight $\Lambda_{1}$ are easily obtained from the ones for highest weight $\Lambda_{0}$ by symmetry of the Dynkin diagram, so we start by only discussing the latter. As $V_{s_{1}}\left(\Lambda_{0}\right)=\mathbf{C}$ we only need to consider Weyl group elements of the form $w_{N}=\cdots s_{0} s_{1} s_{0}$ ( $N$ factors). 
Proposition 3.1. Let $\mu_{N}$ be the weight distribution of the Demazure module $V_{w_{N}}\left(\Lambda_{0}\right)$. Then,

$$
\begin{array}{ll}
\mathrm{E}_{\mu_{N+1}}\left[a^{2}\right]=\mathrm{E}_{\mu_{N}}\left[a^{2}\right]+\frac{N\left(N^{2}+N+2\right)}{16}+2 \operatorname{Cov}_{\mu_{N}}\left(b,(a-b)^{2}\right) & \text { for odd } N, \\
\mathrm{E}_{\mu_{N+1}}\left[b^{2}\right]=\mathrm{E}_{\mu_{N}}\left[b^{2}\right]+\frac{N^{2}(N+1)}{16}+2 \operatorname{Cov}_{\mu_{N}}\left(a,(a-b)^{2}\right) & \text { for even } N .
\end{array}
$$

Proof. We only consider the case of odd $N$, the other case being similar. Then $\mu_{N+1}=D_{1} \mu_{N}=D_{1} D_{0} \mu_{N-1}$. We will use Sanderson's formula for the real character of a Demazure module [10, which implies that the distribution of $a-b$ with respect to $\mu_{N}$ is given by

$$
\left(a-b+\frac{N-1}{2}\right)_{*} \mu_{N}=2^{N} B\left(N, \frac{1}{2}\right),
$$

where $B\left(N, \frac{1}{2}\right)$ is the binomial distribution for $N$ trials with success probability $\frac{1}{2}$.

By unwinding Demazure's character formula [2, Lemma 3.6], we obtain

$$
\mathrm{E}_{\mu_{N+1}}\left[a^{2}\right]=\frac{\mu_{N}(\Gamma)}{\mu_{N+1}(\Gamma)}\left(2 \mathrm{E}_{\mu_{N}}\left[a^{2}\right]+2 \operatorname{Cov}_{\mu_{N}}\left(a^{2}, a-b\right)\right) .
$$

As $\frac{\mu_{N}(\Gamma)}{\mu_{N+1}(\Gamma)}=\frac{1}{2}$ by 3.1 ,

$$
\begin{aligned}
\mathrm{E}_{\mu_{N+1}}\left[a^{2}\right] & =\mathrm{E}_{\mu_{N}}\left[a^{2}\right]+\operatorname{Cov}_{\mu_{N}}\left(a^{2}, a-b\right) \\
& =\mathrm{E}_{\mu_{N}}\left[a^{2}\right]+\operatorname{Cov}_{\mu_{N}}\left((a-b)^{2}+2 a b-b^{2}, a-b\right) \\
& =\mathrm{E}_{\mu_{N}}\left[a^{2}\right]+\underbrace{\operatorname{Cov}_{\mu_{N}}\left((a-b)^{2}, a-b\right)}_{=: A_{N}}+2 \underbrace{\operatorname{Cov}_{\mu_{N}}(a b, a-b)}_{=: B_{N}}+\underbrace{\operatorname{Cov}_{\mu_{N}}\left(b^{2}, a-b\right)}_{=: C_{N}} .
\end{aligned}
$$

From (3.1) it is straightforward to show that $A_{N}=\frac{N}{4}$. Note that the symmetry of $\mu_{N}$ gives

$$
C_{N}=\operatorname{Cov}_{\mu_{N}}\left(b^{2}, a-b\right)=0,
$$

which is a straightforward generalization of [2, Lemma 3.7] (our Proposition 5.2 below contains a general formulation of this phenomenon). Finally, for $B_{N}$, write

$$
B_{N}=\operatorname{Cov}_{\mu_{N}}(a b, a-b)=\mathrm{E}_{\mu_{N}}[a b(a-b)]-\mathrm{E}_{\mu_{N}}[a b] \mathrm{E}_{\mu_{N}}[a-b] .
$$

Then

$$
\begin{aligned}
\mathrm{E}_{\mu_{N}}[a b(a-b)] & =\mathrm{E}_{\mu_{N}}[((a-b)+b) b(a-b)] \\
& =\mathrm{E}_{\mu_{N}}\left[(a-b)^{2} b\right]+\mathrm{E}_{\mu_{N}}\left[b^{2}(a-b)\right] \\
& \stackrel{3.2}{=} \mathrm{E}_{\mu_{N}}\left[(a-b)^{2} b\right]+\mathrm{E}_{\mu_{N}}\left[b^{2}\right] \mathrm{E}_{\mu_{N}}[a-b],
\end{aligned}
$$

and

$$
\begin{aligned}
\mathrm{E}_{\mu_{N}}[a b] \mathrm{E}_{\mu_{N}}[a-b] & =\mathrm{E}_{\mu_{N}}[((a-b)+b) b] \mathrm{E}_{\mu_{N}}[a-b] \\
& =\left(\mathrm{E}_{\mu_{N}}[(a-b) b]+\mathrm{E}_{\mu_{N}}\left[b^{2}\right]\right) \mathrm{E}_{\mu_{N}}[a-b] \\
& \stackrel{3.2}{=}\left(\mathrm{E}_{\mu_{N}}[a-b] \cdot \mathrm{E}_{\mu_{N}}[b]+\mathrm{E}_{\mu_{N}}\left[b^{2}\right]\right) \mathrm{E}_{\mu_{N}}[a-b] \\
& =\mathrm{E}_{\mu_{N}}[a-b]^{2} \cdot \mathrm{E}_{\mu_{N}}[b]+\mathrm{E}_{\mu_{N}}\left[b^{2}\right] \mathrm{E}_{\mu_{N}}[a-b]
\end{aligned}
$$

yield

$$
B_{N}=\mathrm{E}_{\mu_{N}}\left[(a-b)^{2} b\right]-\mathrm{E}_{\mu_{N}}[a-b]^{2} \mathrm{E}_{\mu_{N}}[b]
$$




$$
=\underbrace{\operatorname{Var}_{\mu_{N}}(a-b)}_{\frac{N}{4} \text { by }[3.1]} \underbrace{\mathrm{E}_{\mu_{N}}[b]}_{\frac{(N-1)(N+2)}{8}}+\operatorname{Cov}_{\mu_{N}}\left(b,(a-b)^{2}\right) .
$$

Substituting $A_{N}, B_{N}, C_{N}$ above yields the proposition.

\section{Palindromicity of the StRing Functions}

Let us describe a symmetry property of level 1 Demazure modules, which is crucial for us.

Lemma 4.1. Let $\lambda \in \Lambda_{0}+\mathbf{Z} \alpha_{0}+\mathbf{Z} \alpha_{1}$. Then

$$
\operatorname{dim} V_{w_{N}}\left(\Lambda_{0}\right)_{\lambda}=\operatorname{dim} V_{w_{N}}\left(\Lambda_{0}\right)_{\lambda-S(N, \lambda) \delta},
$$

where

$$
S(N, \lambda)= \begin{cases}\frac{1}{4} N^{2}+(a-b)^{2}(\lambda)-2 a(\lambda) & \text { if } N \text { is even } \\ \frac{1}{4}\left(N^{2}-1\right)+(a-b)^{2}(\lambda)-(a-b)(\lambda)^{6} & \text { if } N \text { is odd } .\end{cases}
$$

Proof. Recall the definition of the bivariate Macdonald polynomials $P_{\nu}\left(z_{1}, z_{2} ; q, t\right) \in$ $\mathbf{Q}(q, t)\left[z_{1}, z_{2}\right]^{S_{2}}$, where $\nu$ is a partition into at most 2 parts (see [6, Chapter VI]). We only use the specializations $P_{\nu}\left(z, z^{-1} ; q, 0\right) \in \mathbf{Q}\left[z^{ \pm}, q^{ \pm}\right]$. If $\nu$ is the one row partition $\nu=(N, 0)$, then [11, Theorem 6 and Theorem 7] gives

$$
\operatorname{ch}\left(V_{w_{N}}\left(\Lambda_{0}\right)\right)=e^{\Lambda_{0}-\left\lfloor\frac{1}{4} N^{2}\right\rfloor \delta} \cdot P_{\nu}\left(e^{\frac{1}{2} \alpha_{1}}, e^{-\frac{1}{2} \alpha_{1}} ; e^{\delta}, 0\right) .
$$

For $N \geq k \geq 0$ and $i \in\{0, \ldots, k(N-k)\}$ let $s_{N, k, i}$ denote the number of lattice paths in $\mathbf{Z}^{2}$ from $(0,0)$ to $(k, N-k)$, where each segment of the path is either $(1,0)$ or $(0,1)$, and such that the area under the path is $i$. As explained in [9], the Gaussian binomial coefficient with parameters $N, k$ in a variable $q$ is

$$
\left[\begin{array}{c}
N \\
k
\end{array}\right]_{q}=\sum_{i=0}^{k(N-k)} s_{N, k, i} q^{i} .
$$

By [3, (3.4)]

$$
P_{\nu}\left(z, z^{-1} ; q, 0\right)=\sum_{k=0}^{N}\left[\begin{array}{l}
N \\
k
\end{array}\right]_{q} z^{2 k-N} .
$$

Combining (4.1) and 4.2 we obtain

$$
\operatorname{ch}\left(V_{w_{N}}\left(\Lambda_{0}\right)\right)=e^{\Lambda_{0}-\left\lfloor\frac{1}{4} N^{2}\right\rfloor \delta} \cdot \sum_{k=0}^{N}\left[\begin{array}{l}
N \\
k
\end{array}\right]_{e^{\delta}} e^{(2 k-N) \frac{1}{2} \alpha_{1}} .
$$

By considering the point reflection in $\left(\frac{k}{2}, \frac{N-k}{2}\right)$, it follows immediately from the above description that the Gaussian binomial coefficients are palindromic, $\left[\begin{array}{c}N \\ k\end{array}\right]_{q}=q^{k(N-k)}\left[\begin{array}{c}N \\ k\end{array}\right]_{q^{-1}}$ and our lemma follows by straightforward computation.

\section{The STRETCHING TRICK}

We want to establish a proper recurrence relation based on the equations described in Proposition 3.1. This section describes how the palindromicity of the string functions allows us to compute the values of $\operatorname{Cov}_{\mu_{N}}\left(\cdot,(a-b)^{2}\right)$ at $b$ and $a$, respectively.

The following two propositions follow directly from the definitions. 


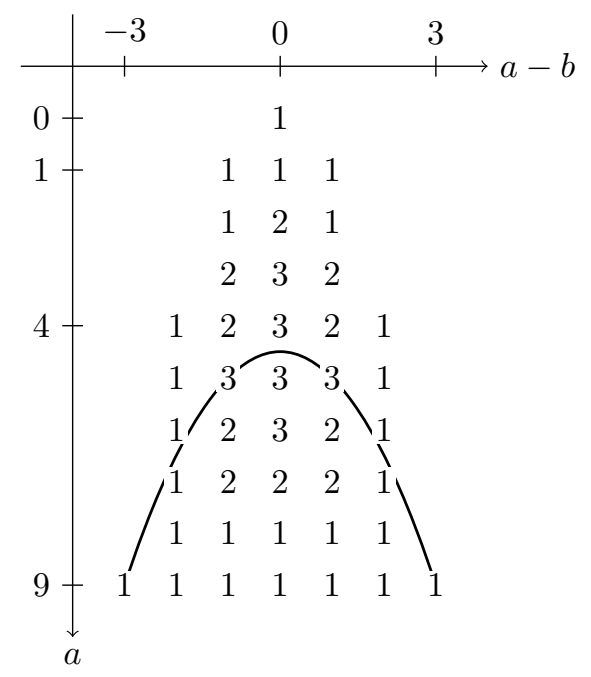

FIGURE 3. Weight distribution $\mu_{6}$ of $V_{w_{6}}\left(\Lambda_{0}\right)=V_{\left(s_{1} s_{0}\right)^{3}}\left(\Lambda_{0}\right)$ and the parabola of the string symmetry points.

Proposition 5.1. Let $\mu \in \operatorname{Meas}\left(\mathbf{R}^{2}\right)$. Let $X, Y: \mathbf{R}^{2} \rightarrow \mathbf{R}$ be the projection on the first and second component, respectively. Let $q: \mathbf{R}^{2} \rightarrow \mathbf{R}^{2}$ be given by $q(x, y)=\left(x^{2}, y\right)$. Then

$$
\operatorname{Cov}_{\mu}\left(X^{2}, Y\right)=\operatorname{Cov}_{q_{*} \mu}(X, Y) .
$$

By saying that $s: \mathbf{R}^{2} \rightarrow \mathbf{R}^{2}$ is a reflection at $H_{1}$ along $H_{2}$ we mean that $H_{1}$ is the 1-eigen space and $H_{2}$ is the (-1)-eigen space of $s$. A measure $\mu \in \operatorname{Meas}\left(\mathbf{R}^{2}\right)$ is said to be symmetric with respect to $s$ if $s_{*} \mu=\mu$.

Proposition 5.2. Let $X, Y: \mathbf{R}^{2} \rightarrow \mathbf{R}$ be the projection on the first and second component, respectively. Let $\mu \in \operatorname{Meas}\left(\mathbf{R}^{2}\right)$ be symmetric at $\{Y=0\}$ along $\{X=0\}$. Then $\operatorname{Cov}_{\mu}(X, Y)=0$.

Now we are ready to exploit the symmetry of the string functions associated with the Demazure module $V_{w_{N}}\left(\Lambda_{0}\right)$.

Lemma 5.3. Let $\mu_{N}$ be the weight distribution of the Demazure module $V_{w_{N}}\left(\Lambda_{0}\right)$ supported on the lattice $\Gamma_{0}=\Lambda_{0}+\mathbf{Z} \alpha_{0}+\mathbf{Z} \alpha_{1}$. Define coordinates $X, Y: \Gamma_{0} \rightarrow \mathbf{R}$ as follows: Let

$$
X=\left\{\begin{array}{ll}
a-b-\frac{1}{2} & \text { if } N \text { is odd, } \\
a-b & \text { if } N \text { is even, }
\end{array} \quad Y= \begin{cases}b-\frac{N^{2}-2}{8} & \text { if } N \text { is odd, } \\
a-\frac{N^{2}}{8} & \text { if } N \text { is even. }\end{cases}\right.
$$

Let $q: \Gamma_{0} \rightarrow \Gamma_{0}$ such that $X(q(\lambda))=X(\lambda)^{2}$ and $Y(q(\lambda))=Y(\lambda)$ for all $\lambda \in \Gamma_{0}$. Then

$$
\operatorname{Cov}_{q_{*} \mu_{N}}(X-2 Y, X)=0 .
$$

While reading the proof, see Figure 3 and 4 for an illustration.

Proof. If $N$ is odd, then by Lemma 4.1 the strings in $\mu_{N}$ are symmetric around

$$
b=\frac{1}{2}\left(\frac{1}{4}\left(N^{2}-1\right)+(a-b)^{2}-(a-b)\right)
$$




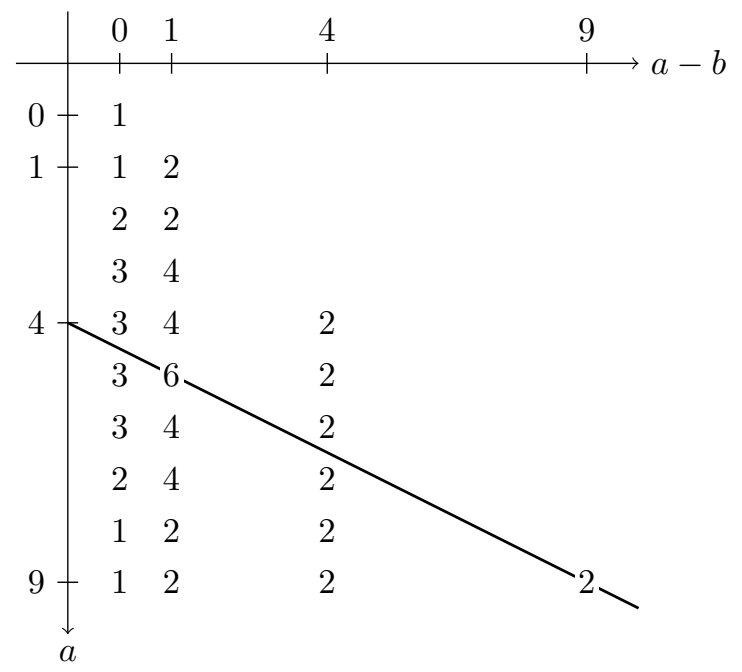

FIgURE 4. Stretched weight distribution $q_{*} \mu_{6}$ of $V_{w_{6}}\left(\Lambda_{0}\right)=$ $V_{\left(s_{1} s_{0}\right)^{3}}\left(\Lambda_{0}\right)$ and the line of the string symmetry points.

$$
\begin{aligned}
& =\frac{1}{2}\left(\frac{1}{4}\left(N^{2}-1\right)+\left(a-b-\frac{1}{2}\right)^{2}-\frac{1}{4}\right) \\
& =\frac{1}{8}\left(N^{2}-2\right)+\frac{1}{2}\left(a-b-\frac{1}{2}\right)^{2} .
\end{aligned}
$$

In other words, they are symmetric around $Y=\frac{1}{2} X^{2}$. If $N$ is even, then by Lemma 4.1 the strings in $\mu_{N}$ are symmetric around

$$
a=\frac{1}{2}\left(\frac{1}{4} N^{2}+(a-b)^{2}\right)=\frac{1}{8} N^{2}+\frac{1}{2}(a-b)^{2} .
$$

In other words, they are symmetric around $Y=\frac{1}{2} X^{2}$. In both cases, the string midpoints in $\mu_{N}$ are $\left(x, \frac{1}{2} x^{2}\right)$ in coordinates $X, Y$, so the string midpoints in $q_{*} \mu_{N}$ are $\left(x^{2}, \frac{1}{2} x^{2}\right)$. Hence $q_{*} \mu_{N}$ is symmetric at $\{X-2 Y=0\}$ along $\{\mathrm{X}=0\}$. The lemma follows by Proposition 5.2.

By formulating Lemma 5.3 in terms of the weight distribution of $V_{w_{N}}\left(\Lambda_{0}\right)$ via Proposition 5.1 we obtain:

Corollary 5.4. Let $\mu_{N}$ be the weight distribution of the Demazure module $V_{w_{N}}\left(\Lambda_{0}\right)$. If $N$ is odd, then

$$
\operatorname{Cov}_{\mu_{N}}\left((a-b)^{2}-(a-b)-2 b,(a-b)^{2}-(a-b)\right)=0 .
$$

If $N$ is even, then

$$
\operatorname{Cov}_{\mu_{N}}\left((a-b)^{2}-2 a,(a-b)^{2}\right)=0 .
$$

Consequently we can now determine the values of $\operatorname{Cov}_{\mu_{N}}\left(\cdot,(a-b)^{2}\right)$ at $b$ and $a$, respectively: 
Lemma 5.5. Let $\mu_{N}$ be the weight distribution of the Demazure module $V_{w_{N}}\left(\Lambda_{0}\right)$. Then,

$$
\frac{N(N-1)}{16}= \begin{cases}\operatorname{Cov}_{\mu_{N}}\left(b,(a-b)^{2}\right) & \text { if } N \text { is odd }, \\ \operatorname{Cov}_{\mu_{N}}\left(a,(a-b)^{2}\right) & \text { if } N \text { is even. }\end{cases}
$$

Proof. By Corollary 5.4 we obtain for odd $N$

$$
\begin{aligned}
\operatorname{Cov}_{\mu_{N}}\left(b,(a-b)^{2}\right)= & \frac{1}{2}\left(\operatorname{Var}_{\mu_{N}}\left((a-b)^{2}\right)-2 \operatorname{Cov}_{\mu_{N}}\left((a-b)^{2}, a-b\right)\right. \\
& \left.+\operatorname{Var}_{\mu_{N}}(a-b)+2 \operatorname{Cov}_{\mu_{N}}(b, a-b)\right),
\end{aligned}
$$

and for even $N$

$$
\operatorname{Cov}_{\mu_{N}}\left(a,(a-b)^{2}\right)=\frac{1}{2} \operatorname{Cov}_{\mu_{N}}\left((a-b)^{2},(a-b)^{2}\right)=\frac{1}{2} \operatorname{Var}_{\mu_{N}}\left((a-b)^{2}\right) .
$$

We know the values on the right-hand sides of both equations. Let us recollect them in either case. If $N$ is odd, we know by [2, Lemma 3.7] that $\operatorname{Cov}_{\mu_{N}}(b, a-b)=0$, and from 3.1 one can deduce

$$
\operatorname{Cov}_{\mu_{N}}\left((a-b)^{2}, a-b\right)=\frac{N}{4} \quad \text { and } \quad \operatorname{Var}_{\mu_{N}}(a-b)=\frac{N}{4} .
$$

Hence,

$$
\operatorname{Cov}_{\mu_{N}}\left(b,(a-b)^{2}\right)=\frac{1}{2}\left(\frac{N(N+1)}{8}-2 \frac{N}{4}+\frac{N}{4}-2 \cdot 0\right)=\frac{N(N-1)}{16} .
$$

If $N$ is even, we again apply (3.1) to derive

$$
\operatorname{Var}_{\mu_{N}}\left((a-b)^{2}\right)=\frac{N(N-1)}{8} .
$$

\section{VARIANCE OF THE DEGReE Distribution}

Proposition 3.1 and Lemma 5.5 immediately give:

Lemma 6.1 (Recurrence relations). Let $\mu_{N}$ be the weight distribution of the Demazure module $V_{w_{N}}\left(\Lambda_{0}\right)$. Then,

$$
\begin{array}{ll}
\mathrm{E}_{\mu_{N+1}}\left[a^{2}\right]=\mathrm{E}_{\mu_{N}}\left[a^{2}\right]+\frac{N^{2}(N+3)}{16} & \text { if } N \text { is odd, } \\
\mathrm{E}_{\mu_{N+1}}\left[b^{2}\right]=\mathrm{E}_{\mu_{N}}\left[b^{2}\right]+\frac{N\left(N^{2}+3 N-2\right)}{16} & \text { if } N \text { is even. }
\end{array}
$$

In order to resolve the recurrence relations, we need to switch between the coordinates $a$ and $b$ depending on the parity of $N$. Therefore, the following version of Lemma 6.1 is more practical.

Lemma 6.2 (Modified recurrence relations). Let $\mu_{N}$ be the weight distribution of the Demazure module $V_{w_{N}}\left(\Lambda_{0}\right)$. Then,

$$
\begin{array}{ll}
\mathrm{E}_{\mu_{N+1}}\left[a^{2}\right]=\mathrm{E}_{\mu_{N}}\left[b^{2}\right]+\frac{N(N+2)(N+3)}{16} & \text { if } N \text { is odd, } \\
\mathrm{E}_{\mu_{N+1}}\left[b^{2}\right]=\mathrm{E}_{\mu_{N}}\left[a^{2}\right]+\frac{N(N+1)(N+2)}{16} & \text { if } N \text { is even. }
\end{array}
$$


Proof. Write $a^{2}-b^{2}=(a-b)(a+b)$ and consider

$$
\operatorname{Cov}_{\mu_{N}}(a-b, a+b)=\mathrm{E}_{\mu_{N}}\left[a^{2}-b^{2}\right]-\mathrm{E}_{\mu_{N}}[a-b] \mathrm{E}_{\mu_{N}}[a+b] .
$$

For odd $N$ we obtain

$$
\begin{aligned}
\mathrm{E}_{\mu_{N}}\left[a^{2}\right] & =\mathrm{E}_{\mu_{N}}\left[b^{2}\right]+\operatorname{Cov}_{\mu_{N}}(a-b, a)+\operatorname{Cov}_{\mu_{N}}(a-b, b)+\mathrm{E}_{\mu_{N}}[a-b] \mathrm{E}_{\mu_{N}}[a+b] \\
& =\mathrm{E}_{\mu_{N}}\left[b^{2}\right]+\frac{N}{4}+0+\frac{1}{2}\left(\frac{1}{2}+2 \frac{(N-1)(N+2)}{8}\right) \\
& =\mathrm{E}_{\mu_{N}}\left[b^{2}\right]+\frac{N(N+3)}{8}
\end{aligned}
$$

by [2, Lemma 3.7, Theorem 4.1], and hence

$$
\mathrm{E}_{\mu_{N+1}}\left[a^{2}\right]=\mathrm{E}_{\mu_{N}}\left[b^{2}\right]+\frac{N(N+3)}{8}+\frac{N^{2}(N+3)}{16}=\mathrm{E}_{\mu_{N}}\left[b^{2}\right]+\frac{N(N+2)(N+3)}{16} .
$$

For even $N$ one similarly derives $\mathrm{E}_{\mu_{N}}\left[b^{2}\right]=\mathrm{E}_{\mu_{N}}\left[a^{2}\right]+\frac{N}{4}$, and consequently

$$
\mathrm{E}_{\mu_{N+1}}\left[b^{2}\right]=\mathrm{E}_{\mu_{N}}\left[a^{2}\right]+\frac{N}{4}+\frac{N\left(N^{2}+3 N-2\right)}{16}=\mathrm{E}_{\mu_{N}}\left[a^{2}\right]+\frac{N(N+1)(N+2)}{16} .
$$

The (modified) recurrence relations give:

Corollary 6.3. Let $\mu_{N}$ be the weight distribution of the Demazure module $V_{w_{N}}\left(\Lambda_{0}\right)$. Then, for $N \geq 1$ we have

$$
\frac{N(N-1)(2 N+5)}{96}=\left\{\begin{array}{l}
\operatorname{Var}_{\mu_{N}}(a) \text { if } N \text { is even, } \\
\operatorname{Var}_{\mu_{N}}(b) \text { if } N \text { is odd. }
\end{array}\right.
$$

Proof. Solving the modified recurrence relations in Lemma 6.2 yields

$$
\begin{aligned}
\mathrm{E}_{\mu_{N}}\left[a^{2}\right] & =\frac{1}{16} \sum_{i=0}^{\frac{N}{2}-1} 2 i(2 i+1)(2 i+2)+\frac{1}{16} \sum_{j=0}^{\frac{N}{2}-1}(2 t+1)(2 t+3)(2 t+4) \\
& =\frac{N\left(3 N^{3}+10 N^{2}+9 N-10\right)}{192}
\end{aligned}
$$

for even $N$, and

$$
\begin{aligned}
\mathrm{E}_{\mu_{N}}\left[b^{2}\right] & =\frac{1}{16} \sum_{i=0}^{\frac{N-1}{2}} 2 i(2 i+1)(2 i+2)+\frac{1}{16} \sum_{j=0}^{\frac{N-3}{2}}(2 t+1)(2 t+3)(2 t+4) \\
& =\frac{(N-1)\left(3 N^{3}+13 N^{2}+10 N-12\right)}{192}
\end{aligned}
$$

for odd $N$. Hence, for even $N$ we get

$$
\begin{aligned}
\operatorname{Var}_{\mu_{N}}(a) & =\mathrm{E}_{\mu_{N}}\left[a^{2}\right]-\mathrm{E}_{\mu_{N}}[a]^{2} \\
& =\frac{N\left(3 N^{3}+10 N^{2}+9 N-10\right)}{192}-\left(\frac{N(N+1)}{8}\right)^{2} \\
& =\frac{N(N-1)(2 N+5)}{96} .
\end{aligned}
$$

Similarly, for odd $N$,

$$
\operatorname{Var}_{\mu_{N}}(b)=\mathrm{E}_{\mu_{N}}\left[b^{2}\right]-\mathrm{E}_{\mu_{N}}[b]^{2}
$$




$$
\begin{aligned}
& =\frac{(N-1)\left(3 N^{3}+13 N^{2}+10 N-12\right)}{192}-\left(\frac{(N-1)(N+2)}{8}\right)^{2} \\
& =\frac{N(N-1)(2 N+5)}{96} .
\end{aligned}
$$

\section{Covariance of the Weight Distribution}

For a distribution $\mu \in \operatorname{Meas}_{\mathrm{c}}\left(\mathbf{Z}^{2}\right)$ and coordinates $X, Y: \mathbf{Z}^{2} \rightarrow \mathbf{Z}$ we define the covariance matrix of $X$ and $Y$ with respect to $\mu$ to be the $2 \times 2$ matrix

$$
\left(\begin{array}{ll}
\operatorname{Cov}_{\mu}(X, X) & \operatorname{Cov}_{\mu}(X, Y) \\
\operatorname{Cov}_{\mu}(Y, X) & \operatorname{Cov}_{\mu}(Y, Y)
\end{array}\right) .
$$

Theorem 7.1 (Covariance of the weight distribution). Let $j \in\{0,1\}$ and $w \in W^{\text {aff }}$ such that the length $l\left(w s_{j}\right)<l(w)=N$. Then the covariance matrix $\Sigma$ of the degree $\langle-d, \bullet\rangle$ and the finite weight $\left\langle\alpha_{1}^{\vee}, \bullet\right\rangle$ in $V_{w}\left(\Lambda_{j}\right)$ is given by

$$
\Sigma=\left\{\begin{array}{cc}
\left(\begin{array}{cc}
\frac{N(N-1)(2 N+5)}{96} & 0 \\
0 & N
\end{array}\right) & \text { if } N \equiv j \bmod (2), \\
\left(\frac{N(N-1)(2 N+5)}{96}+\frac{N}{4}\right. & \frac{N}{2} \\
\frac{N}{2} & N
\end{array}\right) \quad \text { if } N \neq j \bmod (2) .
$$

Proof. We first consider the case that $N \equiv j \bmod (2)$. The variance of the finite weight follows by (3.1), and the fact that degree and finite weight are uncorrelated follows from the symmetry of the weight distribution. The variance of the degree is given by Corollary 6.3, where for odd $N$ we use the nontrivial automorphism of the Dynkin diagram so switch from $V_{w_{N, 0}}\left(\Lambda_{0}\right)$ to $V_{w_{N, 1}}\left(\Lambda_{1}\right)$.

As $V_{w_{N, 0}}\left(\Lambda_{0}\right)$ corresponds to $V_{w_{N, 1}}\left(\Lambda_{1}\right)$ under the nontrivial automorphism of the Dynkin diagram, the result for $N \not \equiv j \bmod (2)$ follows by a change of coordinates.

For visualization purposes, it is convenient to represent the covariance matrix by the associated covariance ellipse, defined as follows: Let $\mu$ be a measure on $\mathbf{R}^{2}$ with nondegenerate covariance matrix $\Sigma$. Then the covariance ellipse of $\Sigma$ is

$$
S_{\mu}=\left\{x \in \mathbf{R}^{2}: x^{t} \Sigma^{-1} x=1\right\} .
$$

In Figure 1, the covariance ellipses have been translated to be centered at the expected weight.

\section{LAW OF LARGE NUMBERS}

We write $\nu_{N} \stackrel{\mathrm{w}}{\longrightarrow} \nu$ if the sequence of measures $\nu_{N}$ converges weakly to $\nu$ as $N \rightarrow \infty$. We will use the following abstract version of the weak law of large numbers, which can be derived from Chebychev's inequality (see e.g. [1, (5.32)]).

Proposition 8.1. Let $\left(P_{N}\right)$ be a sequence of probability distributions on $\mathbf{R}$ such that $\mathrm{E}\left[P_{N}\right] \rightarrow c \in \mathbf{R}$ and $\operatorname{Var}\left(P_{N}\right) \rightarrow 0$. Then, $P_{N} \stackrel{\mathrm{w}}{\longrightarrow} \delta_{c}$.

Finally,

Theorem 8.2 (Weak law of large numbers). Let $\left(\Lambda^{(k)}\right)$ be a sequence in $\left\{\Lambda_{0}, \Lambda_{1}\right\}$, and $\left(w^{(k)}\right)$ a sequence in $W^{\text {aff }}$ such that $l\left(w^{(k)}\right) \rightarrow \infty$. Let $\tilde{\mu}^{(k)} \in \operatorname{Meas}\left(\mathbf{R}^{2}\right)$ be the joint distribution of the degree and the finite weight in $V_{w^{(k)}}\left(\Lambda^{(k)}\right)$, normalized to a 


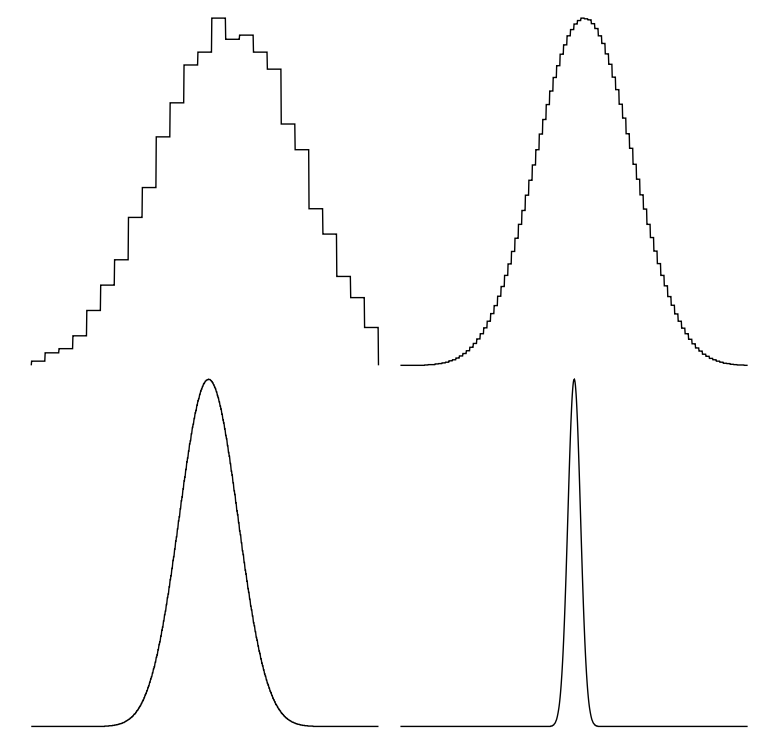

FIGURE 5. Degree distribution of $V_{\left(s_{1} s_{0}\right)^{k}}\left(\Lambda_{0}\right)$ for $k=5,10,25,500$.

probability distribution and rescaled individually in the two coordinates such that $\operatorname{supp}\left(\tilde{\mu}^{(k)}\right)$ just fits into the rectangle $[0,1] \times[-1,1]$. Then , as $k \rightarrow \infty$,

$$
\tilde{\mu}^{(k)} \stackrel{\mathrm{w}}{\longrightarrow} \delta_{\left(\frac{1}{2}, 0\right)} .
$$

See Figure 2 and 5 for an illustration.

Proof. Let $\mu^{(k)} \in \operatorname{Meas}\left(\mathfrak{h}^{*}\right)$ be the weight distribution of $V_{w^{(k)}}\left(\Lambda^{(k)}\right)$, and $\hat{\mu}^{(k)}=$ $\left(-d, \alpha_{0}^{\vee}\right)_{*} \mu^{(k)} \in \operatorname{Meas}(\mathbf{N} \times \mathbf{Z})$ the joint distribution of the degree and the finite weight. We only consider the sequences $\Lambda^{(k)}=\Lambda_{0}$ and $w^{(k)}=w_{2 k, 0}=\left(s_{1} s_{0}\right)^{k}$, as the general case follows easily with small modifications. Then

$$
\begin{aligned}
\max \left\{\langle-d, \lambda\rangle: V_{\left(s_{1} s_{0}\right)^{k}}\left(\Lambda_{0}\right)_{\lambda} \neq 0\right\} & =k^{2}, \\
\max \left\{\left|\left\langle\alpha_{0}^{\vee}, \lambda\right\rangle\right|: V_{\left(s_{1} s_{0}\right)^{k}}\left(\Lambda_{0}\right)_{\lambda} \neq 0\right\} & =k,
\end{aligned}
$$

by [2, Lemma 4.2]. Hence, $\tilde{\mu}^{(k)}$ is the joint distribution of $-\frac{1}{k^{2}} d$ and $\frac{1}{k} \alpha_{1}^{\vee}$ with respect to $\mu^{(k)}$, normalized to a probability distribution. By [2, Theorem 4.5] and Corollary 6.3 we have

$$
\begin{aligned}
\mathrm{E}_{\mu^{(k)}}\left[-\frac{1}{k^{2}} d\right] & =\frac{1}{k^{2}} \mathrm{E}_{\mu^{(k)}}[-d]=\frac{1}{k^{2}} \cdot \frac{k(2 k+1)}{4} \rightarrow \frac{1}{2}, \\
\operatorname{Var}_{\mu^{(k)}}\left(-\frac{1}{k^{2}} d\right) & =\frac{1}{k^{4}} \operatorname{Var}_{\mu^{(k)}}(-d)=\left.\frac{1}{k^{4}} \cdot \frac{N(N-1)(2 N+5)}{96}\right|_{N=2 k} \rightarrow 0 .
\end{aligned}
$$

By Proposition 8.1. $\left(-\frac{1}{k^{2}} d\right)_{*} \mu^{(k)} \stackrel{\mathrm{w}}{\longrightarrow} \delta_{\frac{1}{2}}$. Similarly, $\left(\frac{1}{k} \alpha_{1}^{\vee}\right)_{*} \mu^{(k)} \stackrel{\mathrm{w}}{\longrightarrow} \delta_{0}$, since

$$
\begin{aligned}
\mathrm{E}_{\mu^{(k)}}\left[\frac{1}{k} \alpha_{1}^{\vee}\right] & =\frac{1}{k} \mathrm{E}_{\mu^{(k)}}\left[\frac{1}{k} \alpha_{1}^{\vee}\right]=0, \\
\operatorname{Var}_{\mu^{(k)}}\left(\frac{1}{k} \alpha_{1}^{\vee}\right) & =\frac{1}{k^{2}} \operatorname{Var}_{\mu^{(k)}}\left(\alpha_{1}^{\vee}\right)=\frac{1}{k^{2}} \cdot 2 k \rightarrow 0 .
\end{aligned}
$$


TABLE 1. Conjectural variance of the degree $\operatorname{Var}_{\mu_{N}}(-d)$ in the Demazure module $V_{\left(s_{1} s_{0}\right)^{k}}\left(m \Lambda_{0}\right)$, which is obtained by interpolating values for explicit $N=2 k$. Comparison with the scaling factor for the degree (see [2, Lemma 4.2]) shows that the law of large numbers holds.

\begin{tabular}{c|c|c}
$m$ & $\operatorname{Var}_{\mu_{N}}(-d)$ & $\max \left\{\langle-d, \lambda\rangle: V_{\left(s_{1} s_{0}\right)^{k}}\left(m \Lambda_{0}\right)_{\lambda} \neq 0\right\}$ \\
\hline 2 & $\frac{N(N-1)(4 N+11)}{81}$ & $\frac{1}{2} N^{2}$ \\
3 & $\frac{N(N-1)(34 N+97)}{384}$ & $\frac{3}{4} N^{2}$ \\
4 & $\frac{N(N-1)(52 N+151)}{375}$ & $N^{2}$
\end{tabular}

Let us propose a conjecture about such a concentration in general Demazure modules $V_{w}(\Lambda)$ with analogously normalized and scaled weight distribution $\tilde{\mu}_{w, \Lambda}$. By [2, Corollary 4.3] we know that

$$
\mathrm{E}\left[\tilde{\mu}_{w, \Lambda}\right] \rightarrow \frac{\langle c, \Lambda\rangle+2}{3(\langle c, \Lambda\rangle+1)} \quad \text { as } l(w) \rightarrow \infty .
$$

Consequently,

Conjecture 8.3. Fix a dominant integral weight $\Lambda$ and a sequence $\left(w^{(k)}\right)$ in $W^{\text {aff }}$ such that $l\left(w^{(k)}\right) \rightarrow \infty$. Let $\mu^{(k)} \in \operatorname{Meas}(\mathbf{N} \times \mathbf{Z})$ be the joint distribution of the degree and the finite weight in $V_{w^{(k)}}(\Lambda)$. Let $\tilde{\mu}^{(k)} \in \operatorname{Meas}\left(\mathbf{R}^{2}\right)$ be the distribution obtained from $\mu^{(k)}$ by normalizing to a probability distribution and rescaling the two coordinates individually so that $\operatorname{supp}\left(\tilde{\mu}^{(k)}\right)$ just fits into the rectangle $[0,1] \times[-1,1]$. Then, as $k \rightarrow \infty$,

$$
\tilde{\mu}^{(k)} \stackrel{\mathrm{w}}{\longrightarrow} \delta_{\left(\frac{\langle c, \Lambda\rangle+2}{3(\langle c, \Lambda\rangle+1)}, 0\right)},
$$

where $c=\alpha_{0}^{\vee}+\alpha_{1}^{\vee}$ denotes the canonical central element.

This conjecture is further supported by empirical evidence, see Table 1 . Yet the symmetry property described in $\S 4$ does not hold for higher level Demazure modules. Therefore, the methods employed in this article, in particular §5. do not seem to generalize to this case.

\section{Acknowledgments}

The first author has been supported by the Deutsche Forschungsgemeinschaft, SPP 1388. The second author has been supported by the Deutsche Forschungsgemeinschaft, SFB/TR 12.

\section{REFERENCES}

1. Patrick Billingsley, Probability and measure, third ed., Wiley, 1995.

2. Thomas Bliem and Stavros Kousidis, Expected degree of weights in Demazure modules of $\widehat{\mathfrak{s l}}_{2}$, Transformation Groups (2011), doi:10.1007/s00031-011-9129-6

3. Kazuhiro Hikami, Representation of the Yangian invariant motif and the Macdonald polynomial, J. Phys. A 30 (1997), 2447-2456, doi:10.1088/0305-4470/30/7/023

4. Victor Kac, Infinite-dimensional Lie algebras, third ed., Cambridge University Press, 1990.

5. Shrawan Kumar, Demazure character formula in arbitrary Kac-Moody setting, Invent. Math. 89 (1987), 395-423, doi:10.1007/BF01389086 
6. Ian Macdonald, Symmetric functions and Hall polynomials, second ed., Clarendon Press, 1995.

7. Olivier Mathieu, Formules de Demazure-Weyl, et généralisation du théorème de Borel-WeilBott, C. R. Acad. Sci., Paris, Sér. I 303 (1986), 391-394.

8. __ Formules de caractères pour les algèbres de Kac-Moody générales, Astérisque, no. 159-160, Société Mathématique de France, 1988.

9. George Pólya, Gaussian binomial coefficients and the enumeration of inversions, Proc. Second Chapel Hill Conf. on Combinatorial Mathematics and its Applications, Univ. North Carolina, Chapel Hill, N.C., 1970, pp. 381-384.

10. Yasmine Sanderson, Real characters for Demazure modules of rank two affine Lie algebras, J. Algebra 184 (1996), 985-1000, doi:10.1006/jabr.1996.0294.

11. _ On the connection between Macdonald polynomials and Demazure characters, J. Algebraic Combin. 11 (2000), 269-275, doi:10.1023/A:1008786420650

Thomas Bliem, Department of Mathematics, San Francisco State University, 1600 Holloway Ave, San Francisco CA 94109, United States

E-mail address: bliem@math.sfsu.edu

Stavros Kousidis, Mathematisches Institut, Universität Zu Köln, Weyertal 86-90, 50931 Köln, Germany

E-mail address: st.kousidis@googlemail.com 